\title{
TMPRSS2:ERG—the root of the problem?
}

New data show that TMPRSS2:ERG is expressed at both the RNA and protein levels in prostate cancer stem cells (CSCs), suggesting that current treatments aimed at eliminating luminal cell populations are likely to be inadequate.

In research published in Nature Communications, Polson et al. identified genomic TMPRSS2:ERG in human prostate basal epithelial cultures (derived from prostate CSCs isolated from patient tumour samples) using two-colour break-apart fluorescence in situ hybridization. In virtually all of the fusion-positive cancers, the fusion gene was actively transcribed in the stem cells, implying that it is important for CSC function or survival.

"This is the first demonstration that TMPRSS2:ERG is expressed in prostate CSCs and that colonies founded by these stem cells all contain and express the ERG protein and mRNA," says Norman Maitland, who led the study. "The obvious conclusion is that TMPRSS2:ERG is a cancer-initiating mutation, which acts in stem cells."

In some samples, TMPRSS2:ERG was expressed in CSCs but not in transit-amplifying cells (relatively undifferentiated cells in the basal epithelial compartment), suggesting that fusion gene expression can be switched off. TMPRSS2:ERG expression was restored in the committed basal cell populations of these samples, indicating that expression can be switched from silent to active states (and vice versa) during differentiation.

Polson et al. hypothesize that this effect could be due to an allelic switch between the TMPRSS2 promoter in the fused and unfused alleles. "When stem cells divided to give transit-amplifying cells, the fusion gene was almost always switched off and the unfused allele of TMPRSS2 was switched on," explains Maitland. "This was a true example of asymmetric division (emphasizing the stem-like nature of our cells) and allelic exclusion, which is often seen in embryonal tumours. If allelic expression follows the normal pattern in tissues, there will be patches that express the fused and unfused alleles."

In their conclusions, the authors explain that allelic silencing of activated cellfate-altering CSC loci would promote the accumulation of mutations, maintaining the mutated genotype when oncogeneexpressing daughter cells were destroyed by repair systems and immune responses.

"Our data could explain why certain activated genes are only expressed in a proportion of tumour cells, making it difficult to target all cells with a

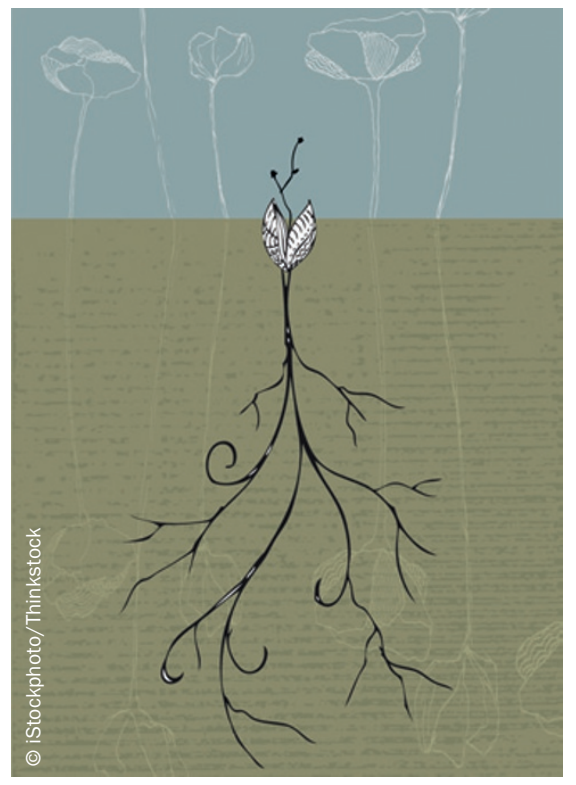

reagent against an activated oncogene," says Maitland. "Silencing might allow mutations to accumulate without any obvious effects on cell morphology, perhaps explaining why most human premalignancies 'appear' with a set of mutations."

Melanie Clyne

Original article Polson, E. S. et al. Monoallelic expression of TMPRSS2/ERG in prostate cancer stem cells. Nat. Commun. doi: 10.1038/ncomms2627 